\title{
artigo
}

Freitas, J.R.; José da Silva, A.; Alves da Silva, J.A.; Ramos, J.R.B.; Vasconcelos Silva, F.M.;

A importância do enfermeiro nas práticas integrativas e complementares no sistema único de saúde

DOI: https://doi.org/10.36489/saudecoletiva.2021v11i63p5376-5389

\section{A importância do enfermeiro nas práticas integrativas e complementares no sistema único de saúde}

The importance of nurses in integrative and complementary practices in the unique health system

La importancia de las enfermeras en las prácticas integrativas y complementarias del sistema único de salud

\section{RESUMO}

As Praticas Integrativas e Complementares (PICS) resgatam os diferentes saberes relacionados ao cuidar em saúde e acontecem de forma concomitante às práticas assistenciais. Objetivo: Desvelar a importância do enfermeiro nas práticas integrativas e complementares no Sistema Único de Saúde Método: Revisão integrativa da literatura realizada através do cruzamento dos descritores padronizados pelo MESH (" Complementary Therapies", "Primare Health Care" e "Public Health Nursing") e seus análogos em Português (DeCS) e em espanhol nas Bases de dados da Medline, Cinahl e Lilacs. 0 processo de seleção dos artigos considerou as recomendações PRISMA e os artigos foram classificados quanto ao nível de evidências através referencial americano AHRQ. Resultado: Foram encontrados 282 artigos, dentre os quais cinco abordaram o tema proposto e foram selecionados para amostra. Conclusão: As PICS favorecem uma maior interação entre enfermeiro-paciente e através da construção de novos saberes desenvolvem estratégias formidáveis de enfrentamento aos problemas de saúde existente.

DESCRITORES: Terapias Complementares. Atenção primária à Saúde. Enfermagem em Saúde Pública.

\section{ABSTRACT}

Integrative and Complementary Practices (PICS) rescue the different knowledge related to health care and happen concurrently with care practices. Objective: To reveal the importance of nurses in integrative and complementary practices in the Unified Health System Method: Integrative literature review carried out by crossing the descriptors standardized by the MESH ("Complementary Therapies", "Primare Health Care" and "Public Health Nursing") and their analogues in Portuguese (DeCS) and Spanish in the Medline, Cinahl and Lilacs databases. The selection process of the articles considered the PRISMA recommendations and the articles were classified according to the level of evidence using the American AHRQ framework. Result: 282 articles were found, of which five addressed the proposed theme and were selected for the sample. Conclusion: PICS favor greater interaction between nurse-patient and, through the construction of new knowledge, develop formidable strategies to face existing health problems.

DESCRIPTORS: Complementary Therapies. Primary Health Care. Public Health Nursing.

\section{RESUMEN}

Las Prácticas Integrativas y Complementarias (PICS) rescatan los diferentes conocimientos relacionados con el cuidado de la salud y suceden de manera concurrente con las prácticas asistenciales. Objetivo: Revelar la importancia del enfermero en las prácticas integradoras y complementarias en el Sistema Único de Salud. Método: Revisión integrativa de la literatura realizada cruzando los descriptores estandarizados por el MESH ("Terapias complementarias", "Atención primaria de salud" y "Enfermería de salud pública") y sus análogos en portugués (DeCS) y español en las bases de datos Medline, Cinahl y Lilacs. El proceso de selección de los artículos consideró las recomendaciones de PRISMA y los artículos se clasificaron según el nivel de evidencia utilizando el marco estadounidense AHRQ. Resultado: Se encontraron 282 artículos, de los cuales cinco abordaron la temática propuesta y fueron seleccionados para la muestra. Conclusión: Los PICS favorecen una mayor interacción enfermera-paciente y, mediante la construcción de nuevos conocimientos, desarrollan formidables estrategias para enfrentar los problemas de salud existentes.

DESCRIPTORES: Terapias complementarias. Atención Primaria de Salud Enfermería de Salud Pública.

RECEBIDO EM: 24/11/2020 APROVADO EM: 07/01/2021

\section{Jadson Rodrigo de Freitas}

Graduando de Enfermagem. Universidade Maurício de Nassau Caruaru, PE.

ORCID: 0000-0002-9913-2104 
Alexsandro José da Silva

Graduando de Enfermagem. Universidade Maurício de Nassau Caruaru, PE.

ORCID: 0000-0001-5315-2575

José Almir Alves da Silva

Enfermeiro. Discente da Pós-graduação latu sensu em Saúde Coletiva da Faculdade Dom Alberto.

ORCID: 0000-0002-8640-9290

\section{Jorge Rosemberg Bezerra Ramos}

Enfermeiro. Especialista em cardiologia e hemodinâmica pelo Instituto Israelita de Ensino e Pesquisa Albert Einstein (IIEP). ORCID: 0000-0001-8628-7417

\section{Fernanda da Mata Vasconcelos Silva}

Doutoranda em enfermagem. Mestre em Enfermagem pela Universidade Federal de Pernambuco (UFPE).

ORCID: 0000-0001-5465-9714

\section{INTRODUÇÃO}

0 Sistema Único de Saúde (SUS) é o modelo adotado no Brasil que confere a população um acesso de forma universal e integral. Tem como objetivo a qualidade de vida através de ações de promoção, prevenção e recuperação da saúde. Em conjunto com o Ministério da Saúde (MS), desenvolve um serviço de maior complexidade sistêmica para garantir a inclusão social através de novos métodos e técnicas ${ }^{1}$.

$\mathrm{Na}$ Primeira Conferência Internacional de Assistência Primária em Saúde (Alma Ata) realizada na Rússia em 1978, foi discutida as primeiras recomendações para a implantação das práticas integrativas e complementares às práticas realizadas pela medicina tradicional. Porém, no Brasil, esse movimento só ganhou força a partir da Oitava Conferência Nacional de Saúde (1986). Em 2003, o MS institui a Política Nacional de Medicina Natural e Práticas Complementares (PMNPC) no SUS e a Portaria $n^{\circ} 971$ de 2006/MS traz o texto atual da Nova Política Nacional de Práticas Integrativas e Complementares (PNPIC) ${ }^{2}$.

As diretrizes de descentralização e participação popular inerentes ao SUS autorizam estados e municípios há uma maior autonomia na definição das suas estratégias e ações voltadas à saúde, possibilitando, dessa forma, implantar as experiências pioneiras como as Práticas Integrativas e Complementares em saúde ${ }^{2}$. Estas se configuram como um mecanismo de vivência e cuidado que auxilia no apoio do equilíbrio físico e emocional. São ações de cuidados transversais, no qual o acolhimento inicia-se na Atenção Básica, que é a principal porta de entrada para o SUS, porém também podendo ser realizadas na média e alta complexidade 3 .

A Medicina Tradicional Chinesa/ Acupuntura, Homeopatias, Fitoterapias, Crenoterapias, Termalismo e Antroposifia aplicada à saúde configuravam as primeiras PICs implantadas no Brasil. Em 2007, já totalizavam quatorze novas práticas desenvolvidas no âmbito do SUS: Arteterapia, Ayurveda, Biodança, Dança Circular, Meditação, Musicoterapia, Naturopatia, Osteopatia, Quiropraxia, Reflexoterapia, Reiki, Shantala, Terapia Comunitária Integrativa Yoga, e em 2018 foram mais dez novas práticas: Aromaterapia Apiterapia, Terapias florais, Terapeutas e Consteladores familiares, Bioenergética, Imposição de mãos Cromoterapia, Geoterapia, Hipnoterapia e Ozonioterapia ${ }^{4}$.

Atualmente, o SUS oferece, de forma integral e gratuita, 29 práticas à população, buscando novas abordagens que estimulem os usuários de forma natural para a prevenção de doenças promovendo uma ótima recuperação da saúde, e prevenção de agravos 5 .

As PICS são reafirmadas como especialidade do Enfermeiro por meio da Resolução COFEN no 581 de 2018, permitindo a eles a excursão das terapias na Saúde Coletiva, Saúde da Criança e do Adolescente, Saúde do Adulto (Saúde do Homem e Saúde da Mulher, Saúde do Idoso, Urgência e
Emergência), assegurando a confiança e o respaldo desses profissionais para atuação nesse cenário, bem como para desenvolver pesquisas na área das PICS em geral ${ }^{6}$.

Esse avanço pode ser compreendido como expressão de um movimento que se identifica com novos modos de aprender a aprender e praticar em saúde, já que as práticas integrativas e complementares em saúde são caracterizadas pela pluralidade interdisciplinar e por linguagens próprias, que em geral se contrapõem à visão tecnicista da saúde que impera no contexto de saúde atual ${ }^{7}$.

A associação das práticas integrativas e complementares à medicina convencional vem ganhando muitos adeptos entre os enfermeiros da atenção primária à saúde ${ }^{8}$. Porém os mesmos precisam ter respaldo técnico-científico para atender ao usuário de forma holística, compreendendo as interações medicamentosas e os efeitos adversos que podem emergir secundário ao emprego de tal prática?.

Esses profissionais tem um foco na saúde, não na doença. Buscam esclarecer aos indivíduos a necessidade de desenvolver tais técnicas terapêuticas alternativas aliadas ao tratamento conservador/ medicamentoso ${ }^{10}$. Porém, na prática clínica, ainda predomina a estrutura do conhecimento científico fracionado posposto por Nightingale em detrimento de uma pactuação profissional-comunidade-rede de apoio, figurando, neste sentido, uma condição excessivamente técnica ao profissional da saúde ${ }^{11}$. 


\section{artigo}

É sabido, portanto, que a incorporação desse modelo de (re)pensar a prática de enfermagem são tidas como fundamentais no contexto contemporâneo. Por desconhecimento de suas contribuiçốes no processo de reestabelecimento da saúde existe ainda, no meio da enfermagem uma percepção distorcida quanto às PICS e tal fato, pode contribuir para desinteresse de diversos profissionais da saúde pelo uso de tais recursos?

Frente a essas circunstâncias, a relevância deste estudo está em desvelar a importância do enfermeiro nas práticas integrativas e complementares no Sistema Único de Saúde, sondando sua capacidade profissional para desenvolver tais práticas com autonomia, em conformidade com a Política Nacional de Práticas Integrativas e Complementares - PNPIC.

\section{MÉTODO}

O estudo utilizou como método de pesquisa a revisão integrativa da literatura. Pautada na prática baseada em evidências, este tipo de revisão busca solucionar os problemas através dos resultados encontrados nas publicações científicas de maior relevância. Envolverá as seguintes etapas: definição do problema de pesquisa, pesquisa nas bases e bancos de dados científicos, avaliação críti-

Figura 1: Fluxograma do processo de seleção do estudo - PRISMA (Preferred Reporting Items for Systematic Reviews and MetaAnalyses) ${ }^{14}$.

Artigos Identificados nas Bases de Dados $(n=282)$

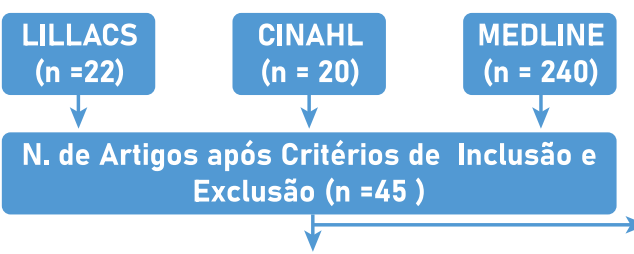

N. de Artigos após leitura dos títulos e resumos $(n=12)$

Artigos selecionados após leitura na integra $(n=05)$

Artigos Incluidos na Síntese (Amostra) $(n=05)$ ca das evidências encontradas e a discussão dos resultados obtidos. Tal prática encoraja a assistência à saúde pautada em conhecimento científico ${ }^{12}$.

Para o levantamento da questão norteadora, empregou-se a estratégia PICO ( $\mathrm{P}$ População: Pacientes assistidos pelo SUS; I - Intervenção: Práticas Integrativas e Complementares em Saúde; C- Comparação: não se aplica; O - Desfecho: Importância do Enfermeiro nas PICS no SUS) ${ }^{13}$. Essa estratégia fundamentada na segmentação da hipótese, visa o levantamento de dados de forma sistematizada ${ }^{13}$. Desta forma, estipulou-se a seguinte questão norteadora da pesquisa: "Qual a importância do enfermeiro nas práticas integrativas e complementares no Sistema Único de Saúde?”

O processo de seleção dos artigos considerou as recomendações PRISMA ${ }^{14}$ (Preferred Reporting Items for Systematic Reviews and MetaAnalyses) (Figura 1). O levantamento dos dados ocorreu no mês de outubro de 2020 nas Bases de Dados da Literatura Latino-Americana e do Caribe em Ciências da Saúde (LILACS), na Cumulative Index to Nursing and Allied Health Literature (CINAHL) e nos Bancos de Dados da Medical Literature Analysis and Retrieval System Online (MEDLINE/ PUBMED). Foram realizados cruzamen-

N. de Artigos Excluídos $(n=237)$

Artigos Excluídos pós leitura na íntegra $(n=40)$ tos dos descritores padronizados pelo Medical Subject Heading (MESH) "Complementary Therapies", "Primare Health Care" e "Public Health Nursing" e seus análogos em Português (DeCS) e em espanhol.

Para definição das informações a serem extraídas dos artigos selecionados e categorização dos estudos utilizou-se busca por pares, com o objetivo de fornecer uma maior credibilidade ao conteúdo da análise. Os descritores foram confrontados de forma pareada e depois em sequências combinadas com prioridade para o descritor "Complementary Therapies", a fim de padronizar os cruzamentos nas bases de dados.

Para seleção dos artigos, foi utilizado como critérios de inclusão: ser artigo original, publicado em português, inglês e espanhol, disponível na íntegra e com recorte temporal de publicação dos últimos cinco anos (2015-2020). Foram excluídos: as teses, as dissertações e as monografias, os editoriais, estudos de caso, as revisões integrativas e as sistemáticas, além das repetições de publicação de estudos em mais de uma base de dados como também os artigos que responderam a questão norteadora do estudo.

Os estudos que compuseram esta revisão foram classificados quanto à prática baseada em evidências, sendo caracterizados de forma hierárquica, utilizando o referencial americano da Agency for Healthcare Reseaech na Quality (AHRQ) que considera o delineamento de pesquisa ${ }^{15}$.

Ressalta-se que a AHRQ classifica a qualidade das evidências em seis níveis: nível 1 : metanálise de múltiplos estudos controlados; nível 2, estudo individual com delineamento experimental; nível 3, estudo com delineamento experimental como estudo sem randomização com grupo único pré e pós-teste, séries temporais ou caso controle; nível 4, estudo com delineamento não experimental como pesquisa descritiva correlacional e qualitativa ou estudo de caso; nível 5, relatórios de casos ou dado obtido de forma sistemática, de qualidade verificável ou dados de avaliação de programas; nível 6, opinião de autoridades respeitáveis baseada na competência clínica ou opinião de comitês de especialistas, incluindo inter- 
pretações de informações de informações não baseadas em pesquisas ${ }^{15}$.

Após leitura e releitura dos artigos selecionados, foi realizada uma categorização em recortes temáticos, com o intuito de descrever e classificar os resultados, evidenciando o conhecimento produzido sobre o tema proposto.

\section{RESULTADOS}

Foram encontrados 289 artigos, dentre os quais cinco abordaram o tema proposto e foram selecionados para compor a amostra final deste estudo. A Medline abrangeu $80 \%$ da quantidade de artigos $(n=4)$, seguida pela
Lilacs (20\%- n=1). A Cinahl não apresentou resultados para a busca realizada.

A especificidade do tema e o uso de artigos originais para composição da amostra funcionaram como uma prerrogativa para quantidade de estudos selecionados. Porém a comunidade científica nacional e internacional esboça a necessidade de novas publicações sobre a temática16-20. A insipiência de artigos pode ser confirmada pelos achados destes estudos que apresentou no ano de 2015, 2018 e 2019 um artigo publicado que respondesse aos objetivos do estudo em cada ano (20\%) e 2020 com dois artigos publicados (40\%). No ano de $2016 \mathrm{e}$ 2017 não foram identificados artigos que se enquadravam nos critérios de inclusão para construção deste estudo.

No quadro 1, as publicações foram organizadas em: autores e ano de publicação, base, nível de evidência, título, objetivo, síntese. Três artigos selecionados para amostra (60\%) estavam publicados em inglês, um artigo $(20 \%)$ em espanhol e um (20\%) em português. Três estudos apresentavam abordagem quantitativa (60\%) e dois abordagem qualitativa (40\%). Neste sentido de acordo com o referencial da AHRQ 60\% das publicações apresentaram nível de evidência 3 e 40\% nível de evidência 4, demonstrando boa qualidade metodológica e redução de viés.

Quadro 1: Síntese dos estudos selecionados para amostra.

\begin{tabular}{|c|c|c|c|}
\hline $\begin{array}{l}\text { AUTOR/ ANO/ } \\
\text { BASE/ NE }\end{array}$ & TÍTULO & OBJETIVO & $\begin{array}{l}\text { IMPORTÂNCIA DO ENFERMEIRO NAS PICS NO } \\
\text { SUS. }\end{array}$ \\
\hline $\begin{array}{l}\text { DALMOLIN; } \\
\text { HEIDEMANN }{ }^{16} \\
2020 \\
\text { Medline/Pubmed } \\
\text { NE: } 4\end{array}$ & $\begin{array}{l}\text { Integrative and complemen- } \\
\text { tary practices in Primary } \\
\text { Care: unveiling health } \\
\text { promotion }\end{array}$ & $\begin{array}{l}\text { Compreender a utilização } \\
\text { das práticas integrativas } \\
\text { e complementares como } \\
\text { ação de promoção da } \\
\text { saúde. }\end{array}$ & $\begin{array}{c}\text { A utilização das PICs pelo enfermeiro configuram-se } \\
\text { como recursos para a promoção da saúde, por meio } \\
\text { da integralidade do cuidado e da redução do uso de } \\
\text { medicamentos. }\end{array}$ \\
\hline $\begin{array}{l}\text { SILVA et al }{ }^{17} \\
2020 \\
\text { Medline/Pubmed } \\
\text { NE:3 }\end{array}$ & $\begin{array}{l}\text { Efeitos da auriculoterapia na } \\
\text { ansiedade de gestantes no } \\
\text { pré-natal de baixo risco }\end{array}$ & $\begin{array}{l}\text { Avaliar os efeitos da auri- } \\
\text { culoterapia nos níveis de } \\
\text { ansiedade em gestantes } \\
\text { atendidas em pré-natal de } \\
\text { baixo risco. }\end{array}$ & $\begin{array}{c}\text { A auriculoterapia pode ajudar a diminuir a } \\
\text { ansiedade em gestantes durante o pré-natal } \\
\text { de baixo risco, sendo uma prática integrativa } \\
\text { e complementar em potencial no âmbito do } \\
\text { Sistema Único de Saúde, tendo o enfermeiro } \\
\text { acupunturista relevante importância nesse } \\
\text { processo. }\end{array}$ \\
\hline $\begin{array}{l}\text { VALIM et } \text { al }^{18} \\
2019 \\
\text { Medline/Pubmed } \\
\text { NE:3 }\end{array}$ & $\begin{array}{l}\text { Auricular acupressure in the } \\
\text { quality of life of women with } \\
\text { breast cancer: a randomized } \\
\text { clinical trial }\end{array}$ & $\begin{array}{l}\text { Avaliar os efeitos da inter- } \\
\text { venção acupressura auricular } \\
\text { na qualidade de vida de } \\
\text { mulheres com câncer de } \\
\text { mama em tratamento qui- } \\
\text { mioterápico, em comparação } \\
\text { com as que não utilizaram a } \\
\text { intervenção. }\end{array}$ & $\begin{array}{l}\text { Acupressura auricular mostrou-se como um método } \\
\text { seguro, eficaz, de baixo custo, sem efeitos colaterais, } \\
\text { facilmente aplicável por enfermeiros treinados. Pode } \\
\text { ser recomendada como terapia complementar no } \\
\text { tratamento do câncer de mama para melhorar a } \\
\text { qualidade de vida dessas mulheres. }\end{array}$ \\
\hline $\begin{array}{l}\text { MELO et al }{ }^{19} \\
2018 \\
\text { Medline/Pubmed } \\
\text { NE: } 3\end{array}$ & $\begin{array}{l}\text { Musical intervention on } \\
\text { anxiety and vital parameters } \\
\text { of chronic renal patients: a } \\
\text { randomized clinical trial }\end{array}$ & $\begin{array}{l}\text { Avaliar o efeito de uma } \\
\text { intervenção musical sobre } \\
\text { a ansiedade e parâmetros } \\
\text { vitais em doentes renais } \\
\text { crônicos em comparação } \\
\text { ao cuidado convencional de } \\
\text { clínicas de hemodiálise }\end{array}$ & $\begin{array}{l}\text { A música apresenta-se como uma potencial inter- } \\
\text { venção de enfermagem para a redução da ansieda- } \\
\text { de-estado durante sessões de hemodiálise }\end{array}$ \\
\hline $\begin{array}{l}\text { LIMA et al }{ }^{20} \\
2015 \\
\text { Lillacs } \\
\text { NE: } 4\end{array}$ & $\begin{array}{l}\text { Uso de terapias integrativas } \\
\text { y complementarias por } \\
\text { pacientes sometidos a la } \\
\text { quimioterapia }\end{array}$ & $\begin{array}{l}\text { Conhecer as terapias inte- } \\
\text { grativas e complementares } \\
\text { utilizadas pelos pacientes } \\
\text { em quimioterapia oncológica. }\end{array}$ & $\begin{array}{l}\text { Apreende-se que uso de terapias integrativas e com- } \\
\text { plementares em oncologia permite a aproximação do } \\
\text { profissional enfermeiro com o paciente em quimiote- } \\
\text { rapia, conhecendo-o em sua complexidade. }\end{array}$ \\
\hline \multicolumn{4}{|l|}{ Fonte: Autores,2020 } \\
\hline
\end{tabular}




\section{DISCUSSÃO}

Para análise e discussão dos artigos com a literatura pertinente foi realizada uma categorização dos estudos na qual emergiu da temática central Importância do enfermeiro nas práticas integrativas e complementares no Sistema Único de Saúde, dois subtópicos: I - Práticas Integrativas e complementares no Sistema Único de Saúde e II - Importancia da utilização Práticas Integrativas e complementares pelo Enfermeiro na promoção a saúde no ambito do SUS.

\section{I - Práticas Integrativas e comple- mentares no Sistema Único de Saúde}

O Sistema Único de Saúde (SUS) trabalha no contexto da integralidade, para a promoção da saúde, porém, enfrenta na prática, muitos desafios. Todavia é inquestionável a ampliação do direito à saúde a toda a população ${ }^{1}$. A Atenção Primária à Saúde (APS), no âmbito do SUS, constitui uma importante porta de entrada assistencial das necessidades de saúde da população. Este nível de atenção está orientado no modelo da Estratégia Saúde da Família (ESF), que prevê uma atuação multiprofissonal à comunidade composta em sua equipe básica por médicos, enfermeiros, técnicos de enfermagem e dentista, além dos agentes comunitários de saúde ${ }^{2,16}$.

Esse modelo assistencial está assegurado por meio da Política Nacional de Atenção Básica (PNAB) 5 . Torna-se cada vez mais perceptível e intenso o ambiente de instabilidade no setor saúde, além das inúmeras tentativas de privatização e institucionalização ${ }^{17}$.

Nesse sentido, compete aos profissionais da área identificar estratégias consolidadas para fortalecimento do SUS, de modo a prestar ao usuário um cuidado humanizado e integral ${ }^{18}$. Uma dessas estratégias é a inserção da Medicina Integrativa como prática complementar em saúde. $\mathrm{Tal}$ inclusão representa uma possibilidade de avanço e integração das diversas práticas em uma mesma rede de atenção, sendo a tomada de decisão pautada de com a necessidade do usuário ${ }^{2}$.

As Praticas Integrativas e Complementares (PICS) resgatam os diferentes saberes relacionados ao cuidar em saúde e acontecem de forma concomitante às práticas clínicas ${ }^{19}$. As PICS são milenares e são oriundas da cultura oriental que prevê um cuidado holístico entre corpo-mente-alma. Ademais, constituem-se em sistemas que promovem a saúde através de técnicas naturais e valorizam a a escuta qualificada, vinculo entre profissional e

O Sistema Único

de Saúde (SUS)

trabalha no contexto

da integralidade,

para a promoção

da saúde, porém,

enfrenta na

prática, muitos

desafios. Todavia

é inquestionável a

ampliação do direito

à saúde a toda a

população. indivíduo e a inter conexão entre os seres, ou seja, as PICS entendem o processo saúde-doença-cuidado de forma ampliada, promovendo a saúde e o autocuidado ${ }^{5,20}$.

A partir da década de 90 , a recorrência de uso das Práticas Integrativas e Complementares em Saúde (PICS) nos tratamentos de doenças crônicas, reabilitação, prevenção de doenças e melhoria da qualidade de vida tem aumentado em proporções mundiais. A Organização Mundial de Saúde (OMS), em 2002 estimulou a regulamentação de tais práticas nos serviços de saúde e em 2006, o Ministério da Saúde (MS), por meio da Portaria no 971/2006, publicou a Política Nacional de Práticas Integrativas e Complementares (PNPIC) no Sistema Único de Saúde (SUS), com o intuito de garantir a integralidade nos serviços de saúde. A partir de então, a oferta e o estímulo ao uso das PICS foi legitimada no SUS e a Atenção Básica à Saúde é um dos principais ambientes para a sua aplicação ${ }^{5}$.

Diante disso, torna-se imperativo analisar o atual cenário de oferta das PICS no país, bem como a acessibilidade e utilização nos serviços de saúde pública. Faz-se necessário observar e desconstruir a postura contra-hegemônica de alguns profissionais e usuários do SUS que ainda tem arraigado uma perspectiva curativista, prescritiva, na qual o verdadeiro cuidar em saúde resume-se em ser atendido pelo médico e realizar exames. A importância das PICS no Cuidado em Saúde deve ser incutida na população desfazendo constructos sociais que foram adquiridos ao longo da vida ${ }^{16-20}$.

II - Importancia da utilização Práticas Integrativas e complementares pelo Enfermeiro na promoção a saúde no ambito do SUS.

O que fundamenta a prática profissional do enfermeiro é o cuidado. Porém, as inferências históricas e culturais influenciam a prática profissional do profissional de enfermagem e por consequência sua tomada de decisão?. Há um movimento de mudança entre o paradigma curativista e medicalizante para o paradigma voltado à prevenção de patologias e promoção à saúde. O que acontece, no âmbito do SUS, é 
que o modelo biologicista ainda prevalece atrelado a prática profissional dificultando assim o processo de ampliação de cuidados à saúde. As PICS incluem abordagens de cuidado que ampliam o olhar sobre o processo saúde-doença e ampliam as possibilidades terapêuticas para os indivíduos ${ }^{4}$.

Considerando a necessidade de ampliar a oferta de PICS nos serviços de saúde, uma das principais estratégias de ações realizadas no âmbito da Política Nacional de Práticas Integrativas e Complementares foram as estratégias de Formação Profissional. No período de 2014 a 2016 mais de 17.500 profissionais de saúde iniciaram processo de formação, mais de 6.500 concluíram e 11.000 estão em curso 5 . Os profissionais de Enfermagem estão entre os que mais se qualificam e conduzem práticas integrativas e complementares, sua atuação está preconizada nas portarias $849 / 2017$ e $702 / 2018$, que ampliam o escopo de práticas ${ }^{17-18}$.

Silva et al (2020) realizou um estudo com objetivo de avaliar os efeitos da auriculoterapia nos níveis de ansiedade em 50 gestantes atendidas em pré-natal de baixo risco de um serviço público do Espírito Santo ${ }^{17}$. O Conselho Federal de Enfermagem estabeleceu e reconheceu por meio da Resolução COFEN no 581 de 2018 a condição técnica do Enfermeiro em realizar essa prática, possibilitando uma apliação dos cuidados de prevenção e promoção da saúde, além da reabilitação das enfermidades ${ }^{6}$.

A assistência de enfermagem em auriculoterapia é uma PICS classificada como um procedimento de baixo custo, fácil aplicação e alto poder de eficácia. Valim (2019) corrobora com Silva et al (2020) quando se trata de acupressura auricular pois, mostrou-se como um método seguro, eficaz, de baixo custo, sem efeitos colaterais, facilmente aplicável por enfermeiros treinados ${ }^{17-18}$.
Nas gestantes estudadas por Silva et al (2020) a aplicação da PIC promoveu a diminuição dos índices de ansiedade e estresse durante o ciclo gestacional ${ }^{17}$. Os resultados também foram satisfatórios nos pacientes oncológicos estudados por Va$\lim$ (2019) e Lima (2015). Os efeitos da intervenção acupressura auricular na qualidade de vida de mulheres com câncer de mama em tratamento quimioterápico no primeiro estudo ${ }^{18}$. E um maior bem-estar, acrescido do senso de autonomia quanto aos processos decisórios sobre seu plano de cuidados foram descritos nos estudos de Lima et al (2015) ao utilizar para pacientes oncológicos as PICS baseadas em plantas medicinais como a homeopatia e a fitoterapia ${ }^{20}$. Apreende-se que uso de PICS em oncologia e obstetrícia permite a aproximação do profissional enfermeiro com o paciente, conhecendo-o em sua complexidade.

Melo et al (2018) identificou que o enfoque na singularidade do ser traz consigo um maior estreitamento de laços entre pacientes e profissionais da saúde e refletiu sobre o efeito de uma intervenção musical sobre a ansiedade e parâmetros vitais em doentes renais crônicos em comparação ao cuidado convencional de clínicas de hemodiálise. A música apresentou-se como uma potencial intervenção de enfermagem para a redução da ansiedade-estado durante sessões de hemodiálise demonstrando mais uma vez a beneficência da inserção das Práticas Integrativas e complementares no SUS ${ }^{19}$.

Implementar de forma efetiva as PICS à pratica cotidiana na atenção básica, configura-se um desafio para gestores, trabalhadores e autoridades sanitárias de saúde ${ }^{21}$. Estudos demonstram que os profissionais relatam dificuldades no manejo de algumas práticas, incipiência de conhecimento sobre outras técnicas e referem precisar de cursos complementares sobre aplicabilidade das PICS no SUS ${ }^{17,19,22}$.

Estudos avaliam o caráter formativo em PICS durante os cursos de graduação em enfermagem e demonstram que há uma imensa lacuna na construção desse novo profissional referente a essa temática. Mudanças e adaptações nas matrizes curriculares são necessárias para formar um profissional capacitado e qualificado, capaz de utilizar todo conhecimento adquirido para prestar um cuidado integral ao seu paciente e fortalecer as ações desenvolvidas pelo sistema de saúde pública brasileira?.

\section{CONCLUSÃO}

O cuidado fundamenta a base da profissão enfermagem. A adição das Praticas Integrativas e Complementares às ações de cuidado já preconizadas possibilita a interação e a troca entre novos saberes. Pautadas na busca de uma assistência holística e integral, as PICS buscam a harmonização do organismo humano e a perfeita sincronia de ações sistêmicas através de mecanismos naturais. Porém, faz-se necessário ampliar o conhecimento sobre suas indicações, métodos e eficácia, pautada em evidências científicas

Este estudo apresenta como relevância demostrar a importância do enfermeiro nas PICs nas ações desenvolvidas no SUS. Acreditamos, dessa forma, contribuir para a formação de profissionais mais capacitados e motivados a implementar às PICs ao cuidado já preconizado pelos órgãos de saúde. As PICS favorecem uma maior interação entre enfermeiro-paciente e através da construção de novos saberes desenvolvem estratégias formidáveis de enfrentamento aos problemas de saúde existente.

\section{REFERÊNCIAS}

1. Telesi JE. Práticas integrativas e complementares em saúde, uma nova eficácia para o SUS. Estudos Avançados.
2016; 30(86): 99-112. DOI: 40142016.00100007
https://doi.org/10.1590/S0103- 


\section{artigo}

Freitas, J.R.; José da Silva, A.; Alves da Silva, J.A.; Ramos, J.R.B.; Vasconcelos Silva, F.M.i

A importância do enfermeiro nas práticas integrativas e complementares no sistema único de saúde

\section{REFERÊNCIAS}

2. Brasil. Ministério da Saúde (BR). Secretaria de Atenção à Saúde. Política nacional de práticas integrativas e complementares no SUS. 2a ed. Brasília; 2015. Disponível em: https://bvsms. saude.gov.br/bvs/publicacoes/politica_nacional_praticas_integrativas_complementares_2ed.pdf

3. Cruz PLB, Sampaio SF. As práticas terapêuticas não convencionais nos serviços de saúde: revisão integrativa. Rev APS 2016; 19(3):483-94. Disponivel em: https://aps.ufjf.emnuvens. com.br/aps/article/view/2594/1054

4. Brasil. Ministério da Saúde (BR). Portaria $n^{\circ} 849$ de 27 de março de 2017. Inclui a Arteterapia, Ayurveda,Biodança, Dança Circular, Meditação, Musicoterapia, Naturopatia, Osteopatia, Quiropraxia, Reflexoterapia, Reiki, Shantala, Terapia Comunitária Integrativa e Yoga à Política Nacional de Práticas Integrativas e Complementares. Diário Oficial da União 2017. Disponível em: $\quad$ http://bvsms.saude.gov.br/bvs/saudelegis/gm/2017/ prt0849_28_03_2017.html

5. Brasil. Ministério da Saúde (BR). Portaria n 702 de 21de março de 2018. Altera a Portaria de Consolidação n²/GM/MS, de 28 de setembro de 2017, para incluir novas práticas na Política Nacional de Práticas Integrativas e Complementares - PNPIC. Diário Oficial da União 2018. Disponivel em: http://bvsms.saude. gov.br/bvs/saudelegis/gm/2018/prt0702_22_03_2018.html

6. COFEN. Conselho Federal de Enfermagem (BR). RESOLUÇÃO COFEN No 581/2018. Atualiza, no âmbito do Sistema Cofen/ Conselhos Regionais de Enfermagem, os procedimentos para Registro de Títulos de Pós - Graduação Lato e Stricto Sensu concedido a Enfermeiros e aprova a lista das especialidades.2018 Disponivel em: http://www.cofen.gov.br/resolucao-cofen-no-581-2018_64383.html

7. Alvin NAT. Práticas integrativas e complementares de saúde no cuidado. Rev Enferm UFSM 2016; 6(1): 4-6 DOI: https://doi. org/10.5902/2179769221571

8. Carvalho RVS, Anjos AMC, Oliveira MM, Silva CAL, Leal SRMD, Oliveira VA, Lima ACG. Práticas integrativas e complementares aplicadas aos trabalhadores de uma Unidade Básica de Saúde: relato de experiência. Rev. Saúde Pública de Mato Grosso do Sul. 2019 2(1-2): 70-76. Disponivel em: http://revista.saude.ms.gov. br/index.php/rspms/article/view/45/58

9. Silva ASP, Feitosa ST. Revisão sistemática evidencia baixo nível de conhecimento acerca da política nacional de práticas integrativas e complementares por parte de gestores e profissionais da saúde. Vitalle 2018;30(1):105-14. DOI: https://doi. org/10.14295/vittalle.v30i1.7491

10. Santiago MECF. Práticas Integrativas e Complementares: a Enfermagem Fortalecendo essa Proposta. Uniciências. 2017; 21(1):50-4. DOI: https://doi.org/10.17921/14155141.2017v21n1p50-54

11. Lopes ACP, Ceolin T, Ceolin S, Lope CV. As contribuições da disciplina "terapias complementares com ênfase em plantas medicinais" na prática profissional dos enfermeiros. Rev Pesqui Cuid Fundam. 2018; 10(3):619-25. DOI: https://doi. org/10.9789/2175-5361.2018.v10i3.619-625
12. Polit DF, Beck CT. Fundamentos da pesquisa em enfermagem: avaliação de evidências para a prática de enfermagem. $7^{\text {a }}$ ed. Porto Alegre: ArtMed; 2011.

13. Santos MARC, Galvão MGA. A elaboração da pergunta adequada de pesquisa. Resid Pediatr., Rio de Janeiro, v. 4, n. 2, p.53-56, ago. 2014. Disponivel em: http://residenciapediatrica. com.br/detalhes/105/a-elaboracao-da-pergunta-adequada-de-pesquisa

14. Moher D, Liberati A, Tetzlaff J, Altman DG, The PRISMA Group. Preferred Reporting Items for Systematic Reviews and Meta-Analyses: The PRISMA Statement. Epidemiol. Serv. Saúde, Brasília, 2015; 24(2): 335-342. DOl: http://doi.org/10.5123/ S1679-49742015000200017

15. Jeanne-Marie Guise, MD. AHRQ Series on Improving Translation of Evidence: Progress and Promise in Supporting Learning Health Systems. The Joint Commission Journal on Quality and Patient Safety 2020; 46:51-52. DOI: https://doi.org/10.1016/j. jcjq.2019.10.008

16. Dalmolin IS, Heidemann ITSB. Integrative and complementary practices in Primary Care: unveiling health promotion. Rev. Latino-Am. Enfermagem 2020; 28: e3277. DOI: https://doi. org/10.1590/1518-8345.3162.3277

17. Silva HL, Almeida MVS, Diniz JSP, Leite FMC, Moura MAV, Bringuente MEO, Brandão-Souza C, et al. Efeitos da auriculoterapia na ansiedade de gestantes no pré-natal de baixo risco. Acta Paul Enferm 2020;33:eAPE20190016. Disponível em: https:// acta-ape.org/article/efeitos-da-auriculoterapia-na-ansiedadede-gestantes-no-pre-natal-de-baixo-risco/

18. Vallim ETA, Marques ACB, Coelho RCFP, Guimarães PRB, Felix JVC, Kalinke LP. Auricular acupressure in the quality of life of women with breast cancer: a randomized clinical trial. Rev. esc. enferm. USP. 2019; 53: e03525. DOI: http://dx.doi.org/10.1590/ s1980-220x2018043603525.

19. Melo GAA, Rodrigues AB, Firmeza MA, Grangeiro ASM, Oliveira PP, Caetano JA. Musical intervention on anxiety and vital parameters of chronic renal patients: a randomized clinical trial. Rev. Latino-Am. Enfermagem. 2018; 26: e2978. DOI: https://doi. org/10.1590/1518-8345.2123.2978.

20. Lima JF, Ceolin S, Pinto BK, Zilmmer JGV, Muniz RM, Schwartz E. Uso de terapias integrativas y complementarias por pacientes sometidos a la quimioterapia. Av Enferm. 2015;33(3):372380. Dispinivel em: http://www.scielo.org.co/pdf/aven/v33n3/ v33n3a05.pdf

21. Soares DP, Coelho AM, Silva LEAS, et al. Política Nacional de práticas integrativas e complementares em saúde: Discurso dos enfermeiros da Atenção Básica. Revista de Enfermagem do Centro Oeste Mineiro. 2019; 9:e3265. DOI: http://dx.doi. org/10.19175/recom.v9i0.3265

22. Almeida JR, Vianini MCS, Silva DM, Meneghin RA, Souza G, Resende MA. O enfermeiro frente às práticas integrativas e complementares em saúde na estratégia de saúde da família. Revista Eletrônica Acervo Saúde 2018; 18 (e77): 1-7. DOI: https://doi. org/10.25248/reas.e77.2019 